\title{
A Case of Vibrio vulnificus Septicemia in a Patient with Gastric Bypass Surgery
}

\author{
Katherine Moll Reitz, Peter David Allen, ${ }^{2}$ Giselle G. Hamad, ${ }^{3}$ and Alain Corcos ${ }^{4}$
}

\begin{abstract}
Background: Vibrio vulnificus is a pathogen found commonly in raw oysters that rarely causes one of two clinical presentations after ingestion: primary septicemia with associated metastatic soft tissue infections or gastroenteritis. Despite the infrequent incidence of the disease, the mortality rate of primary septicemia approaches $50 \%$. In the affected patient population, the organism survives the gastrointestinal tract despite gastric acid release. Most cases of Vibrio vulnificus primary septicemia are found in those with chronic liver disease, hemochromatosis, or immunodeficiency/immunosuppression.

Case Presentation: A 46-year-old Caucasian male with history of a remote Roux-en-Y gastric bypass presented with bilateral lower extremity necrotizing soft tissue infection after ingestion of raw oysters. The patient was emergently debrided and treated with broad-spectrum antibiotics for suspected, and later culture-confirmed, Vibrio vulnificus infection. Despite the patient having none of the well-established risk factors, the patient suffered from multi-system organ failure and disseminated intra-vascular coagulopathy, ultimately resulting in a large ischemic stroke and death.

Conclusions: This report demonstrates mortality of a patient with none of the previously identified risk factors for primary septicemia. We propose that decreased gastric acidity associated with Roux-en-Y gastric bypass may be a risk factor for the development of primary septicemia from Vibrio vulnificus and should be investigated further.
\end{abstract}

$\boldsymbol{V}$ IBRIO VULNIFICUS IS a gram negative bacilli that is part of the natural flora of worldwide salt-water coastal environments. Despite the almost universal presence of the bacteria on raw oysters in the summer months, only approximately 200 infections occur annually in the United States [1]. After the ingestion of infected raw seafood, the opportunistic infection can lead to one of two clinical presentations: primary septicemia or gastroenteritis. A majority of affected patients are immunosuppressed or suffer from chronic liver disease. Ninety-five percent of patients with primary septicemia seek medical attention within seven days of ingesting raw oysters [1]. Patients present most frequently with fevers, chills, nausea, and bacteremia, typically resulting in metastatic soft tissue infections of the lower and occasionally upper extremities. The mortality rate for primary

septicemia is approximately $50 \%$, and prompt diagnosis and treatment is key to survival [2]. Patients require emergent wide surgical debridement and prompt initiation of antibiosis. The U.S. Centers for Disease Control recommends treatment with a third-generation cephalosporin and doxycycline. We report a case of culture-proven primary septicemia secondary to $V$. vulnificus resulting in patient death despite aggressive source control and prompt antibiotic therapy.

\section{Case Presentation}

A 46-year-old Caucasian male presents as a transfer from an outside hospital complaining of $20 \mathrm{~h}$ of sharp, sudden onset, constant and progressive, right worse than left, lower

\footnotetext{
${ }^{1}$ Department of General Surgery, University of Pittsburgh Medical Center, Presbyterian Hospital, Pittsburgh, Pennsylvania.

${ }^{2}$ Department of General Surgery, ${ }^{4}$ Department of Trauma and Acute Care Surgery, University of Pittsburgh Medical Center, Mercy Hospital, Pittsburgh, Pennsylvania.

${ }^{3}$ Minimally Invasive Bariatric and General Surgery, University of Pittsburgh Medical Center, Presbyterian and Magee-Women's Hospital, Pittsburgh, Pennsylvania.

(C) Katherine Moll Reitz et al. 2016; Published by Mary Ann Liebert, Inc. This Open Access article is distributed under the terms of the Creative Commons License (http://creativecommons.org/licenses/by/4.0), which permits unrestricted use, distribution, and reproduction in any medium, provided the original work is properly credited.
} 
extremity pain with associated red--purple discoloration and superficial bullae. His medical history was notable for laparoscopic Roux-en-Y gastric bypass for weight loss with a $150 \mathrm{~cm}$ retrocolic roux limb $10 \mathrm{y}$ prior resulting in a 100pound weight loss, coronary artery disease status post-stent placement, and a recent umbilical hernia repair with mesh. He denied any trauma to his lower extremities, insect bites, recent travel, or any other associated symptoms including fevers or diarrhea. The patient had no history of chronic liver disease or immunosuppression, and the pre-operative workup for his umbilical hernia repair included a normal comprehensive metabolic panel with liver function testing. Furthermore, the patient had no visible liver pathology on multiple prior laparoscopic surgical interventions. The patient's family noted that three days prior to arrival, he had ingested a large volume of seafood including raw oysters at a restaurant in southwestern Pennsylvania.

On arrival, the patient was in severe distress requiring emergent intensive care unit admission, where he deteriorated rapidly requiring mechanical ventilation and pharmacologic hemodynamic support. Bilaterally, his toes were dusky with associated blotchy red--purple skin changes and hemorrhagic bullae diffusely covering the right, and to a lesser extent, the left lower extremities primarily below the knee (Fig. 1). He was started empirically on vancomycin, piperacillintazobactam, and clindamycin. He was debrided emergently down to fascia and a four-compartment fasciotomy was completed revealing intact musculature. The hemorrhagic fluid from the bullae (Fig. 2) as well as presenting blood cultures would eventually confirm Vibrio vulnificus primary septicemia likely secondary to the consumption of raw oysters three days prior.

Despite aggressive and repeated surgical debridement and early transition to ceftriaxone, doxycycline, ciprofloxacin,

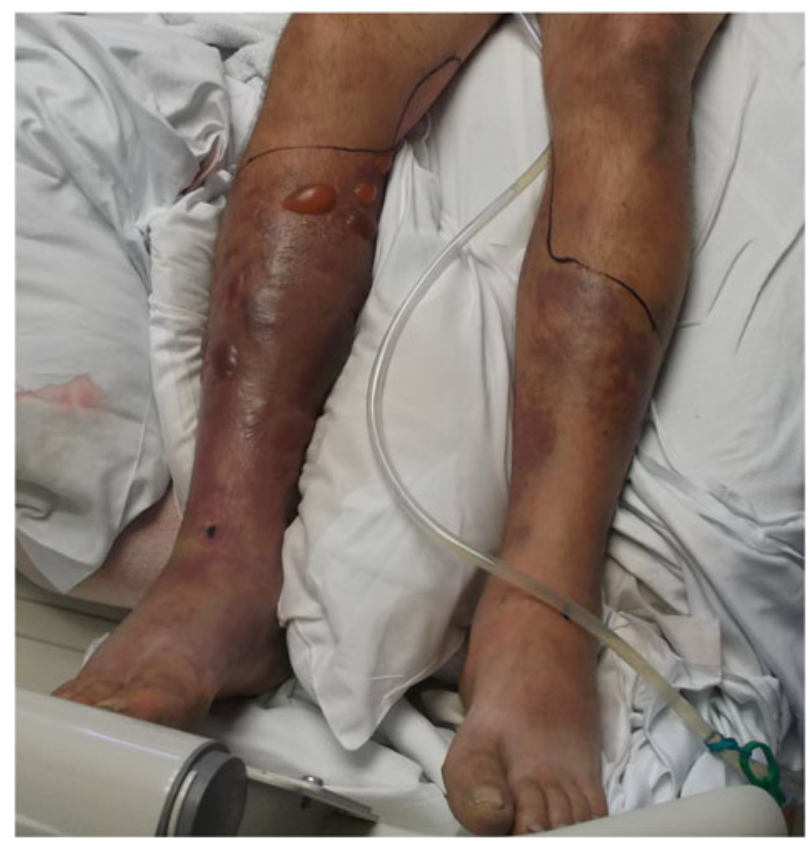

FIG. 1. Initial presentation of bilateral lower extremities with red-purple discoloration and associated hemorrhagic bullae.

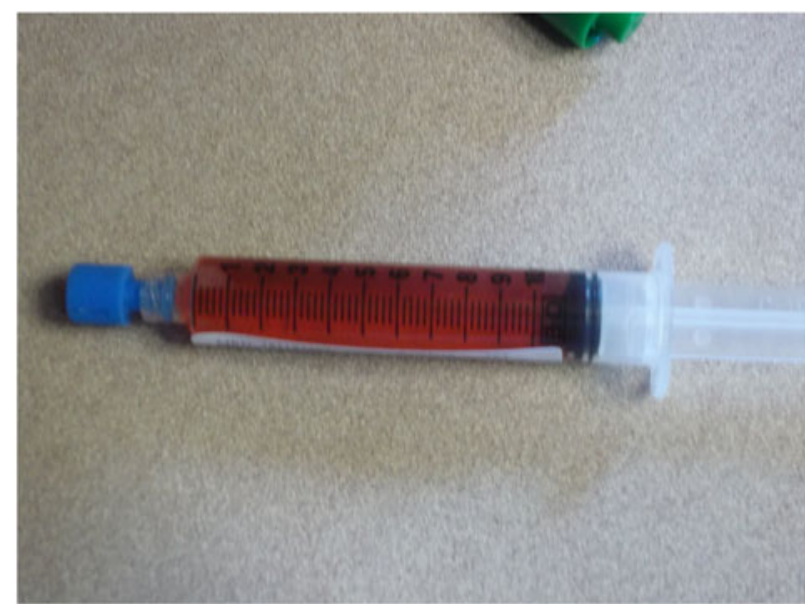

FIG. 2. Fluid expressed from hemorrhagic bullae, final culture positive for Vibrio vulnificus.

and clindamycin, the patient's condition progressed with new skin lesions present in the bilateral distal upper extremities. Laboratory workup confirmed disseminated intra-vascular coagulation and the patient was later found to have a massive left middle cerebral artery stroke. After further deterioration, comfort measures only were implemented, and he died on hospital day six.

\section{Discussion and Review of the Literature}

Vibrio vulnificus is responsible for more than $95 \%$ of seafood-related deaths in the United States [3]. Of those who develop primary septicemia, which has approximately a $50 \%$ mortality rate, more than $95 \%$ have at least one risk factor including chronic liver disease, immunocompromised status, hemochromatosis, diabetes mellitus, or age older than 60 years [2]. The two most well established risk factors are immunocompromised status and chronic liver disease, increasing the risk of primary septicemia by more than 80 -fold [2].

The patient discussed had no known risk factors for the development of primary septicemia, however, he did have a history of obesity treated surgically with Roux-en-Y gastric bypass. Although obesity can be associated with such liver dysfunction as non-alcoholic steatohepatitis, this patient had normal laboratory testing, normal visualization of his liver, and significant weight loss after Roux-en-Y gastric bypass, leading us to consider other risk factors for infection including the anatomic and metabolic alterations inherent to gastric bypass surgery.

The host's first defense from $V$. vulnificus after ingestion of the bacteria is the acidic environment of the stomach. In vitro, when $V$. vulnificus is exposed to normal gastric contents for 20 min there is a resulting 4-log decrease in surviving bacterial cells [4]. Furthermore, animal models demonstrate that an increased gastric $\mathrm{pH}$ (a less acidic environment) can lead to an increased risk of infection with a lower bacterial load required for clinically significant infection [5-7]. Therefore, the bacteria's ability to survive the acidic environment of the stomach is key to its systemic dissemination via entrance into the blood stream through the distal small intestine and cecum [8]. 
Clinical studies have confirmed the widely accepted principle that there is a clinically and statistically significant decrease in basal and peak production of gastric acid in patients after Roux-en-Y gastric bypass $[9,10]$. Therefore, by means of his Roux-en-Y gastric bypass, this patient would have increased gastric $\mathrm{pH}$, decreasing his ability to destroy ingested bacteria and ultimately increasing his risk of systemic infection. We therefore propose Roux-en-Y gastric bypass as a potential risk factor to the development of primary septicemia after the ingestion of $V$. vulnificus even in the absence of other previously published risk factors.

\section{Conclusion}

We recommend that patients who have decreased gastric acid, specifically those with a history of Roux-en-Y gastric bypass, should be considered at higher risk of primary septicemia after ingestion of Vibrio vulnificus. Therefore, although direct correlation cannot be established based on this case report, we recommend that patients who have undergone Roux-en-Y gastric bypass avoid ingestion of raw oysters to prevent $V$. vulnificus primary septicemia, particularly in the summer months in which the risk of being exposed to inoculated oysters is the greatest. The gastrointestinal system after Roux-en-Y gastric bypass is less acidic leading to decreased host protection and increasing the risk that $V$. vulnificus will survive into small intestine. Here the pathogen gains entrance into the blood stream causing primary septicemia, which carries a $50 \%$ mortality rate.

\section{Author Disclosure Statement}

No competing financial interests exist.

\section{References}

1. Scallan E, Hoekstra RM, Angulo FJ, et al. Foodborne illness acquired in the United States-Major pathogens. Emerg Infect Dis 2011;17:7-15.

2. Jones MK, Oliver JD. Vibrio vulnificus: Disease and pathogenesis. Infect Immun 2009;75:1723-1733.

3. Baker-Austin C, McArthur JV, Lindell AH. Multi-site analysis reveals widespread antibiotic resistance in the marine pathogen Vibrio vulnificus. FEMS Microbiol Ecol 2009;57:151-159.

4. Koo J, DePaola A, Marshall DL. Antacid increases survival of Vibrio vulnificus and Vibrio vulnificus phage in gastrointestinal model. Appl Environ Microbiol 2001;67:28952902.

5. Koenig KL, Mueller J, Rose T. Vibrio vulnificus-Hazard on the half shell. West J Med 1991;155:400-403.

6. Akhondi H, Lopez AG. Necrotizing fasciitis secondary to Vibrio vulnificus. J Med Cases 2014;5:650-652.

7. Koo J, DePaola A, Marshall DL. Effect of stimulated gastric fluid and bile on survival of Vibrio vulnificus and Vibrio vulnificus phage. J Food Prot 2000;63:1665-1669.

8. Horseman MA, Surani S. A comprehensive review of $V i$ brio vulnificus: An important cause of severe sepsis and skin and soft-tissue infections. Int J Infect Dis 2011;15: e157-e166.

9. Smith CD, Herkes SB, Behrns KE, et al. Gastric acid secretion and vitamin B12 absorption after vertical Roux-en-Y gastric bypass for morbid obesity. Ann Surg 1993;218: 91-96.

10. Behrns KE, Smith CD, Sarr MG. Prospective evaluation of gastric acid secretion and cobalamin absorption following gastric bypass for clinically severe obesity. Dig Dis Sci 1994;39:315-320.

Address correspondence to:

Dr. Katherine Moll Reitz

University of Pittsburgh Medical Center,

Presbyterian Hospital

Department of General Surgery 200 Lothrop Street

Pittsburgh, PA 15213

E-mail: reitzkm2@upmc.edu

Cite this article as: Reitz KM, Allen PD, Hamad GG, Corcos A. (2016) A case of Vibrio vulnificus septicemia in a patient with gastric bypass surgery. Surgical Infections Case Reports 1:1, 100-102, DOI: 10.1089/ crsi.2016.0025 